Wright State University

CORE Scholar

\title{
Navigating the Decision Space: Shared Medical Decision Making as Distributed Cognition
}

\author{
Katherine D. Lippa \\ Wright State University \\ Markus Alexander Feufel \\ Wright State University \\ F. Eric Robinson \\ Wright State University \\ Valerie L. Shalin \\ Wright State University - Main Campus, valerie.shalin@wright.edu
}

Follow this and additional works at: https://corescholar.libraries.wright.edu/psychology

Part of the Psychology Commons

\section{Repository Citation}

Lippa, K. D., Feufel, M. A., Robinson, F. E., \& Shalin, V. L. (2016). Navigating the Decision Space: Shared Medical Decision Making as Distributed Cognition. Qualitative Health Research, 27 (7), 1035-1048. https://corescholar.libraries.wright.edu/psychology/442

This Article is brought to you for free and open access by the Psychology at CORE Scholar. It has been accepted for inclusion in Psychology Faculty Publications by an authorized administrator of CORE Scholar. For more information, please contact library-corescholar@wright.edu. 


\title{
Navigating the Decision Space: Shared Medical Decision Making as Distributed Cognition
}

Qualitative Health Research

$1-14$

(C) The Author(s) 2016

Reprints and permissions:

sagepub.com/journalsPermissions.nav DOI: $10.1177 / 1049732316665347$ qhr.sagepub.com

\author{
Katherine D. Lippa ${ }^{\text {* }^{*}}$, Markus A. Feufel ${ }^{2^{*}}$, F. Eric Robinson', \\ and Valerie L. Shalin'
}

\begin{abstract}
Despite increasing prominence, little is known about the cognitive processes underlying shared decision making. To investigate these processes, we conceptualize shared decision making as a form of distributed cognition. We introduce a Decision Space Model to identify physical and social influences on decision making. Using field observations and interviews, we demonstrate that patients and physicians in both acute and chronic care consider these influences when identifying the need for a decision, searching for decision parameters, making actionable decisions Based on the distribution of access to information and actions, we then identify four related patterns: physician dominated; physician-defined, patient-made; patient-defined, physician-made; and patient-dominated decisions. Results suggests that (a) decision making is necessarily distributed between physicians and patients, (b) differential access to information and action over time requires participants to transform a distributed task into a shared decision, and (c) adverse outcomes may result from failures to integrate physician and patient reasoning. Our analysis unifies disparate findings in the medical decision-making literature and has implications for improving care and medical training.
\end{abstract}

\section{Keywords}

doctor-patient communication and relations; cognition; decision making; chronic disease; Multiple Sclerosis; emergency care; qualitative; grounded theory; North America

\section{Introduction}

Health care research and policy increasingly emphasize shared decision making (Hibbard \& Greene, 2013; Thille \& Russell, 2010). However, research on shared decision making is often limited to examining isolated choice points and/or factors facilitating or hindering patient involvement, without addressing the decision-making process itself (Bernabeo \& Holmboe, 2013; Légaré \& Witteman, 2013). This approach reflects a limited understanding of the reasoning involved in shared decision making and of the factors that shape the distribution of clinical cognition among patients and health professionals.

As physical, organizational, and institutional work occur along a clinical illness trajectory (Strauss, Fagerhaugh, Suczek, \& Wiener, 1995), so too clinical cognition is an ongoing process that occurs throughout the duration of care. Thus, a cognitive trajectory occurs in parallel with a patient's illness trajectory. Medical decisions occur precisely when the current trajectory needs to be altered, prompting physicians and patients to share information and coordinate action (Elwyn et al., 2014; Epstein, 2013). To elaborate the ensuing, interrelated processes, we employ the paradigm of distributed cognition.
Distributed cognition locates thought as an emergent property of people interacting with other actors and the environment rather than a process inherently restricted to individual minds. This paradigm motivates the analysis of decision making as a social, physical, and cognitive process that integrates multiple actors' perspectives (Cowley \& Vallée-Tourangeau, 2013; Hutchins, 1995). Researchers have examined distributed cognition among medical practitioners (Engeström, Engeström, \& Kerosuo, 2003; Feufel, Robinson, \& Shalin, 2011; Hazlehurst, McMullen, \& Gorman, 2007). However, to date, little is known about how patients and health professionals engage in distributing clinical cognition (Lippa \& Shalin, 2015).

To illuminate how this distributed cognitive system influences medical decision making, we first review established

\footnotetext{
'Wright State University, Dayton, Ohio, USA

${ }^{2}$ Charité-Universitätsmedizin Berlin, Germany

*Joint first authors
}

Corresponding Author:

Katherine D. Lippa, Dept of Psychology, Wright State University, 3640 Colonel Glenn Hwy, Dayton OH 45435, USA.

Email: lippa2@gmail.com 
approaches to understanding medical decision making. Then, we present a Decision Space Model to classify the influences that determine and initiate decision needs and direct the unfolding decision-making processes. We conclude this introduction by expanding the Decision Space Model to accommodate the inherent distribution of functions in the context of shared decision making.

\section{Approaches to Understanding Medical Decision Making}

Researchers have examined medical decision making using both quantitative and qualitative methods. On the quantitative side, laboratory studies have analyzed how individuals - mostly medical professionals or studentsprocess information to make decisions given predefined decision points and assumed medical solutions/evaluation criteria (e.g., Croskerry, 2009). For example, cognitive science researchers have made extensive comparisons between the diagnostic accuracy of physicians using simple decision rules that are adapted to answer specific diagnostic questions and domain-independent normative statistical models (e.g., Phang, Ravani, Schaefer, Wright, \& McLaughlin, 2015; Reyna \& Lloyd, 2006). However, neither heuristic, intuition-based, nor more elaborate hypothetical-deductive decision models (Custers, 2013; Schwartz \& Elstein, 2009) shed light on how decision points are identified, how relevant decision-making parameters are selected, or the definition of appropriate criteria for a "good" clinical decision.

Quantitative research focused on shared medical decision making typically examines a small number of factors (e.g., health literacy, diagnosis, intervention programs) in relation to specific outcomes (e.g., satisfaction with care, accurate risk assessment, disease status; Ghane, Huynh, Andrews, Tabuenca, \& Sweeny, 2014; Hibbard \& Greene, 2013). By contrast, qualitative studies mainly examine the role of socio-cultural phenomena such as interpersonal dynamics, discourse, or power relations in decision making (Corbin, 1998; Roter \& Hall, 2006), but attend less to information flow and associated cognitive processes.

We argue that the process focus pertaining to most quantitative approaches and the impact of socio-cultural influences on decision making emphasized by most qualitative approaches are complementary. Both are necessary to understand how decision points are identified and how relevant decision-making parameters are selected for making "good" clinical decisions. Clinical care involves parameters that are not well replicated in laboratory settings, and, in fact, contradicts some basic experimental assumptions: In clinical care, decision points and outcome criteria are not given and situations are uncontrolled and unpredictable, in part, because multiple players influence the decision process (Klein \& Wright,
2016). Clinical decision making is a part of an ongoing trajectory complicated by fluctuating preferences, contextual constraints, and ambiguous task requirements (Broadstock \& Michie, 2000). These contextual factors form a socio-cognitive landscape that is as inextricably linked to decision-making processes and outcomes as topography is to nautical navigation (Hutchins, 1995). We argue that when integrated into this socio-cognitive landscape, (shared) decision-making processes and related challenges such as misdiagnoses and non-adherence may be better understood (Barber, 2002; Cameron, Leventhal, \& Leventhal, 1993). We begin by identifying cognitive, physical, and social influences on the decisionmaking process.

\section{The Decision Space Model}

We suggest the Decision Space Model in Figure 1 to specify and classify the physical, psychological, and social influences that shape individual decision making. The model was originally developed to describe physicians' decision making in acute care settings (Feufel, 2009). The lower half of the figure is consistent with constructs in the classical literature on problem solving (Simon \& Newell, 1971). The upper half of the figure is consistent with classical constructs in social science (Kleinman, 1980).

The Decision Space Model treats individual cognitive processes, environmental constraints (state space), and opportunities for action (action space) as functionally related but conceptually separable components of a distributed decision-making process. The state space consists of the properties of the task environment, where states evolve as a consequence of both human action and natural processes over time. The model differentiates physical constraints, such as resources, technology, and probabilistic relationships between symptoms and diseases, as limits on the range of possible states. In medical settings, such physical considerations include the patient's physiological status and the availability of resources to manipulate a patient's status such as biomedical technology and treatment options. Values enable a distinction between desirable states (potential goals) from undesirable states (potential problems). Values include personal or professional preferences related to culturally defined notions of health care, illness, and quality of life considerations.

The action space encompasses participants' ability to generate desirable and acceptable states (e.g., March \& Olsen, 2006). The range of feasible actions is limited by personal capacities, such as psychomotor or cognitive abilities. The range of acceptable action is limited by adopted norms, socially accepted rules governing action, including informally defined social roles as well as formalized institutional policies. In medical settings, this 


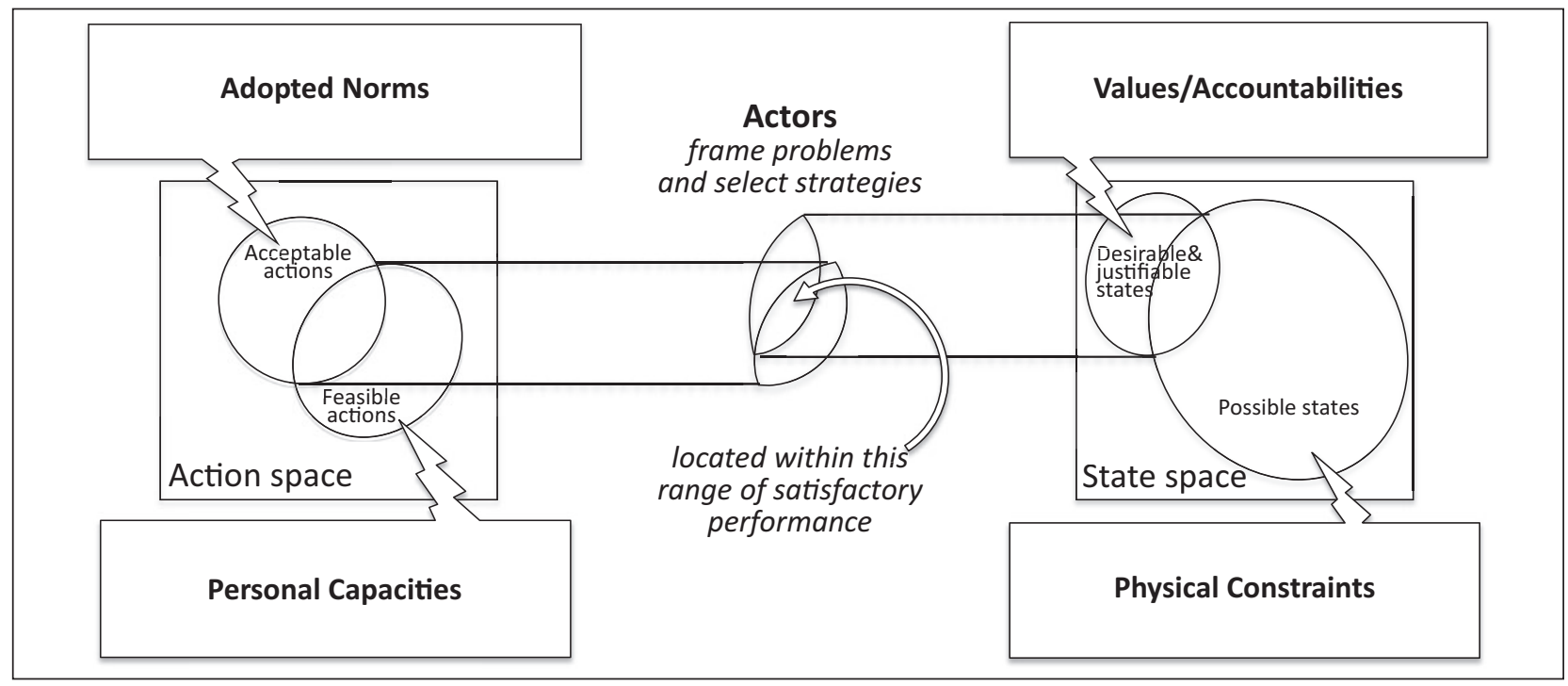

Figure I. The decision space for a single actor.

may refer to the practice of limiting diagnostic procedures to the level required to make and justify a treatment decision (Shalin \& Bertram, 1996) or admit or discharge a patient (Feufel, 2009). In combination with the prevailing state space, adopted norms determine the availability, selection, and sequencing of justifiable actions.

The Decision Space Model associates the need for decisions with potential changes in the trajectory for clinical cognition and care. The need for a decision may result from natural progression along an illness trajectory or from the identification of undesirable and potentially remediable current states. Decision makers then select and combine parameters of the state space with options from the action space to consider in making the decision. Finally, evaluation of these options according to a system of values and norms yields the decision.

\section{Toward an Understanding of Shared Medical Decision Making}

Critically, in medical decision making, individual actors typically only have partial access to the full state and action spaces. Physicians and patients are working on the same problem, but their understandings of the decision space may differ. In the state space, the physician depends upon the patient for information about her state of health (e.g., symptoms and comorbidity) and personal values. Similarly, the patient depends upon the physician for biomedical information, such as symptom-disease contingencies. In the action space, the physician depends upon the patient to adhere to treatment, whereas the patient depends upon the physician for access to treatments that require medical expertise or authority. In the action space, norms and personal capacities shape each actor's contribution to treatment. This inherent interdependence implies that shared decision making between physician and patient is a distributed cognitive process comparable with that between medical professionals (Engeström et al., 2003; Epstein, 2013; Feufel et al., 2011). Therefore, decision making must draw on the perspectives of both physician and patient to result in an effective cooperative system (Malone \& Crowston, 1994).

Below, we support an extended version of the Decision Space Model to cover shared decision-making processes involving both physicians and patients (see Figure 2). Analysis focuses on the identification of patterns of decision making and how they reflect the sharing of the decision-making process in the Decision Space Model. Discussion concerns ramifications of these findings for extending conventional, theoretically driven notions of medical decision making and its practical applications.

\section{Method}

This study draws on two data sets focused on physicianpatient interactions, one collected in Emergency Departments (Feufel, 2009) and the other collected in a clinic specializing in Multiple Sclerosis (Lippa \& Shalin, 2015). Initially separate data analyses revealed findings that were mutually reinforcing. Preliminary investigation of the Multiple Sclerosis data focused on the decisionmaking process and yielded four decision patterns, differentiating the particular contributions of physicians and patients in medical decision making. Preliminary analysis of the Emergency Department data focused on influences between environmental factors and medical decisions 


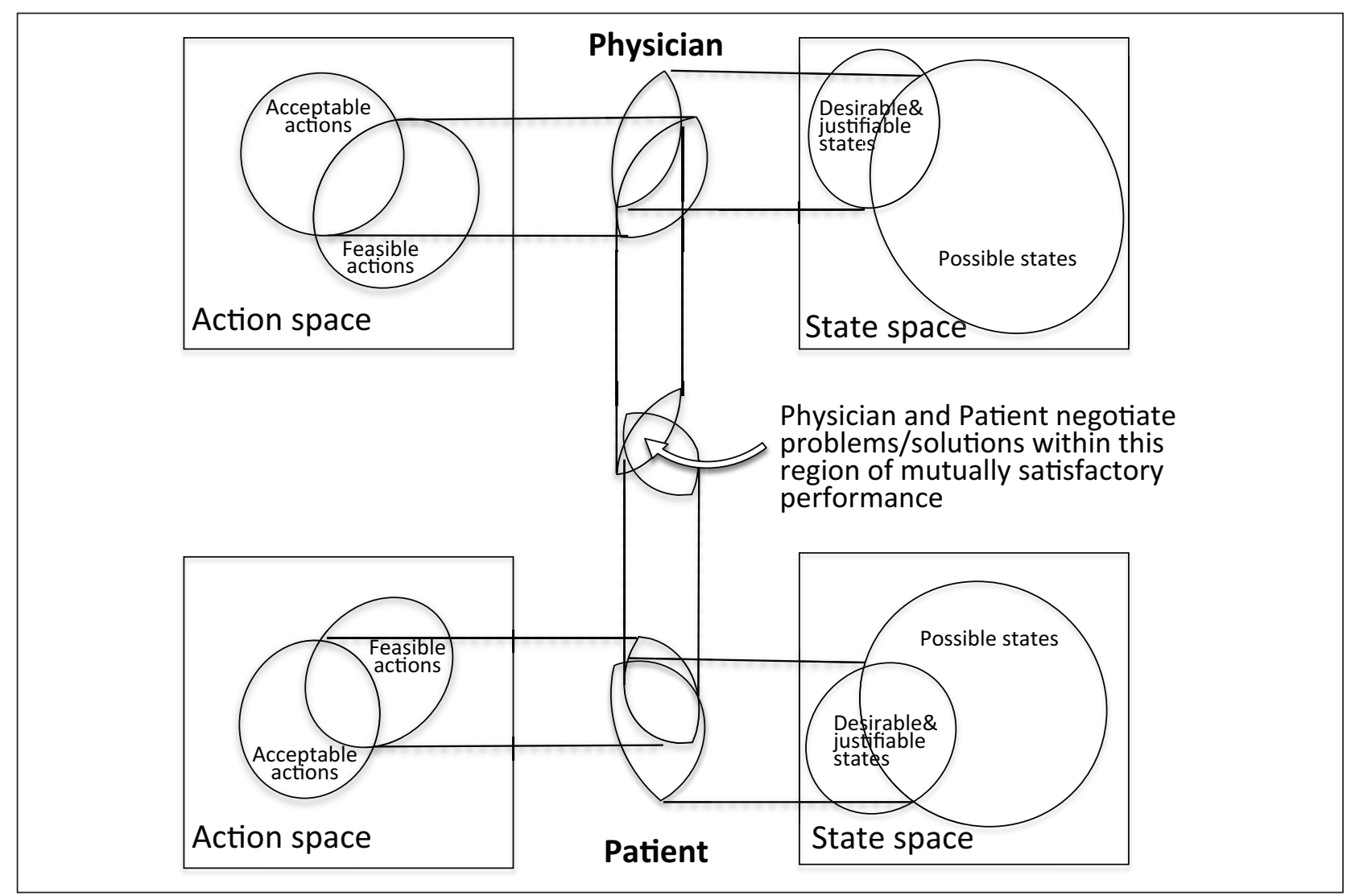

Figure 2. The integration of physician and patient perspectives to a mutually acceptable decision space.

and led to the development of the Decision Space Model. Because both studies used field observations and interviews for similar conceptual reasons but looked at different forms of medical care, this article uses both data sets as a means of data triangulation to support the generalizability of results and identifies the common contextual factors that influence the decision processes presented. Below, we describe the conceptual perspective for both studies, the separate processes of data collection and initial analyses, and the common final analysis.

\section{Conceptual Perspective}

Both studies originated from a concern for the psychological considerations that inform the design of efficient work systems and technology. The research was motivated by the belief that design recommendations depend on understanding how medical decisions are made in context. Such an understanding requires a combination of knowledge about medical practice and a broad theoretical conceptualization of medical decision making. However, much of the prevailing theory assumed both pre-specified decision points and evaluation criteria. Accordingly, the researchers chose to collect data through field observations and interviews to capture physicians' decision making in context when interacting with patients, to ground a theoretical model of medical decision making.

\section{Data Collection}

Both studies used participant observation during patientphysician interactions and conversations with informants in the field (medical and clerical staff). The researchers attempted to keep their role as unobtrusive as possible to minimize influence on the interaction. However, all the participants were aware of the researcher's presence and interest in "reasoning processes," creating potential for altering interactions. All consent procedures were approved by institutional review boards (IRBs) at the researchers' home university and the medical facilities where data were collected.

The Multiple Sclerosis data were collected through a specialty care center at a large, Midwestern medical school. Two board-certified neurologists (with 11 and 17 years of experience) and 16 patients (three men and 13 women) participated. Four participants had a high school level education, seven were college educated, three had a post-graduate education, and two did not wish to discuss their educational background. Patients represented a wide range of experience with Multiple Sclerosis, from newly 
diagnosed to having lived with the disease for decades. Physician and patient participants provided written consent. Approximately 65 hours were spent making observations. Data collection included audio recording and/or detailed field notes depending upon the comfort of the participants. To provide as rich a description as possible, the examples below are drawn from audio recordings. Within 14 days of the initial observations, follow-up interviews were conducted with all patients including general questions and focused discussion of problems and decisions from the clinical session. These interviews facilitated the initial analysis by Lippa and Shalin (2015) in providing additional contextual information, clarifying the patient's intentions during and reaction to the clinical interaction. The taped clinical sessions and interviews were transcribed using literary transcription.

The Emergency Department data were gathered in two teaching hospitals associated with the Medical School of a mid-size, Midwestern university: a larger urban hospital and a smaller suburban hospital. The larger urban hospital served a largely uninsured, racially and ethnically mixed population, with a relatively high proportion of lower socio-economic status patients. The patient population at the smaller suburban hospital was generally insured and predominantly Caucasian, with a larger proportion of higher socio-economic status patients. At each Emergency Department, three attending and six resident physicians were followed during a work shift (approximately 10 hours) balanced across shifts and days of the week, summing up to approximately 160 hours of observations. Attending experience ranged from 5 to 31 years, with a mean of 13.2 years. The resident sample consisted of two in each of the first, second, and third years of residency. During each shift, the observed physicians saw between 10 and 28 patients. Given time constraints and to avoid disrupting care, the IRB approved only asking physicians to provide written consent, whereas patients were asked for their consent verbally prior to observation. For the same reason, demographic information could not be systematically collected from the patients. Conversations between physicians and patients as well as with the observer were logged by hand and later transcribed. Notes captured the semantic content of each conversational turn and reflected the participants' word choices.

\section{Initial Data Analyses}

Data from the Multiple Sclerosis clinic were analyzed first, based on the principles of grounded theory (Strauss $\&$ Corbin, 2008). Initial, open coding identified decision making as a core category. All the data involving decisions were microanalyzed for indications of underlying decision-making processes. This analysis included identifying concepts relating to what information was present and how it was used, factors affecting the ultimate decision, and roles adopted by physicians and patients. Connections between the identified concepts revealed four common patterns of decision making depending on the distribution of information, action, and cognitive activities across patient and physician.

The Emergency Department data were initially coded with respect to environmental cues used for making decisions. The initial conceptual distinctions related to singular cues such as medical history, patient preferences, or resource availability. Based on discussions with subject matter experts, these cues were then grouped into two functionally related categories: cues pointing to problematic features of the situation and cues referring to actionable solutions. ${ }^{1}$ Selective coding of instances based on this categorization led to an initial version of the Decision Space Model as a way to locate the observed influences on decision making (Feufel, 2009).

Discussions among the authors suggested that the decision patterns identified in the Multiple Sclerosis clinic data complemented the Decision Space Model identified from the Emergency Department data and vice versa. This prompted the researchers to selectively recode the Emergency Department data based on the decision patterns and re-interpret the Multiple Sclerosis data in light of the Decision Space Model. Comparative analysis of selected decisions from both data sets and associated discussions by the authors helped to refine the conceptualization of the Decision Space Model and the observed decision patterns.

\section{Final Data Analysis}

A final analysis focused on close examination of decision making and discourse in both settings. Individual examples from Multiple Sclerosis management and the Emergency Department were selected to illustrate the decision-making patterns identified. They were selected because they clearly articulated cognitive, discursive, interpersonal, and environmental characteristics that were common to decisions of a particular pattern. The examples are representative in that they include common processes, but often contain higher than average ambiguity or conflict in the decision parameters prompting direct articulation of decision-making efforts.

\section{Results}

\section{Decision-Making Patterns}

In both chronic and acute disease management situations, physicians and patients had access to different portions of the decision space. This led to decision patterns that varied from primarily individual to completely distributed. 
Table I. Patterns of Distributed Medical Decision Making.

\begin{tabular}{|c|c|c|c|c|}
\hline & $\begin{array}{l}\text { Identification of } \\
\text { Decision Point }\end{array}$ & $\begin{array}{l}\text { Definition of } \\
\text { Parameters }\end{array}$ & Final Decision & Characteristic Situations \\
\hline $\begin{array}{l}\text { Physician- } \\
\text { dominated }\end{array}$ & Physician responsibility & Physician responsibility & Physician responsibility & $\begin{array}{l}\text { Decision is highly technical and/or } \\
\text { urgent and requires minimal patient } \\
\text { action }\end{array}$ \\
\hline $\begin{array}{l}\text { Physician-defined, } \\
\text { patient-made }\end{array}$ & $\begin{array}{l}\text { Either actor may be } \\
\text { responsible }\end{array}$ & Physician responsibility & Patient responsibility & $\begin{array}{l}\text { Decision is predicated on biomedical } \\
\text { information but requires patient } \\
\text { action }\end{array}$ \\
\hline $\begin{array}{l}\text { Patient-defined, } \\
\text { physician-made }\end{array}$ & $\begin{array}{l}\text { Either actor may be } \\
\text { responsible }\end{array}$ & Patient responsibility & Physician responsibility & $\begin{array}{l}\text { Decision is dependent upon case } \\
\text { history and/or phenomenological } \\
\text { information but requires physician } \\
\text { action }\end{array}$ \\
\hline Patient-dominated & Patient responsibility & Patient responsibility & Patient responsibility & $\begin{array}{l}\text { Decisions are dependent upon patient } \\
\text { evaluation of the state space and do } \\
\text { not require physician action (i.e., } \\
\text { self-care, self-medication) }\end{array}$ \\
\hline
\end{tabular}

That is, physicians and patients each took responsibility for some or all stages of the decision process: identification of the need for a decision, elaboration of parameters, and final choice. The specific roles adopted by patients and physicians depended upon each participant's access to information about the state space and available actions. Table 1 summarizes the decision-making patterns found and when they typically occur.

In some cases, physicians and patients each dominated decisions, taking responsibility for all stages of the decision-making process. Nonetheless, the role of the other was often at least implicitly considered. In other cases, the decision tasks were split between the physician and patient. One actor identified the need for a decision and established decision-relevant parameters whereas the other made the final choice. The analysis below examines each of the decision-making patterns in detail. Parallel examples from Multiple Sclerosis management and the Emergency Department are provided for each decision type as convergent evidence.

\section{Physician-Dominated Decisions}

Physician-dominated decisions correspond most closely to the decision-making context assumed by cognitively oriented theories related to heuristic decision making (e.g., Marewski \& Gigerenzer, 2012) or hypotheticaldeductive decision making (Schwartz \& Elstein, 2009). Physician-dominated decisions occurred in both settings when the decision need and process were predicated on specialized biomedical knowledge of disease states and the physicians' action space. Some physician-dominated decisions were completely unilateral with no provision to inform or include the patient in the decision. This was especially true when physicians followed discipline-prescribed normative protocols with no complicating factors (e.g., patients presenting with chest pain should have an electrocardiogram [EKG]). However, in some physiciandominated decisions, complicating factors required consideration of the patient's perspective.

In the management of Multiple Sclerosis, a chronic condition, decisions made by physicians alone were typically found at the end of the clinical encounter concerning the formulation of a plan for care (Roter \& Hall, 2006). As part of this conclusion, physicians frequently made unilateral decisions about topics that were routine or highly technical, especially whether and when to send the patient for testing (i.e., magnetic resonance imaging [MRIs], bloodwork). The following example illustrates this process:

\footnotetext{
Physician: I am just debating. Should we repeat the MRIsee where you're at? Or just not do anything and wait? Like I said, it's a little bit difficult to proceed. Because if we were going to know that you can get pregnant in the next two three months there is no reason to even do the MRI. And then again if you are going to be trying to get pregnant we're not going to start your medication anyway. The only thing would be like if the MRI were tremendously bad, what I don't expect, and then we could consider starting you on medication, wait? Otherwise, we're going to try you again to get pregnant after you go off the medication. That's the only thing I am debating right now. But I think that we'll probably just wait to see, you know, how it goes.
}

Here, medical norms within the action space indicate a routine MRI, but the physician identifies a combination of physical constraints (medication that is contraindicated for pregnancy) and values (patient's goal of becoming pregnant) that oppose this norm. This conflict prompts the physician to explore other parameters within the state space (probability of pregnancy and disease status) and how they are linked to possible actions (a diagnostic MRI 
and medical intervention). Only after exploring these parameters does the physician make a final decision.

The exclusivity of the physician's decision-making process here is logical. Only she has the technical expertise to evaluate most of the parameters and weigh whether a test is medically indicated in light of the patient's values. Although the physician does all the cognitive work in this case, she shifts from a first person singular construction to a first person plural. Her use of the first person singular demonstrates that she is in charge of the decision process ("I am debating"), but she shifts to a plural construction when sampling options from her action space ("Otherwise we're going to try you again to get pregnant when you go off the medication") thereby including the patient. This pronoun shift indicates that this physician considers the decision as part of a trajectory of care that reflects patient involvement.

In the acute care Emergency Department setting, physician-dominated decisions occurred mainly when patients required immediate care, when they were incapacitated and their families unavailable, or when subsequent action did not depend on patient preferences. Nevertheless, Emergency Department physicians often considered and vocalized at least part of the clinical reasoning behind their decisions to inform patients about what actions were to be taken and why they were justified. This type of discourse provided the patient/family with access to information defining the patient's current state and associated care alternatives. In response, patients often voiced preferences for certain options based on their values, which the physician could then use to further specify next steps. Consider the following decision about recurrent chest tightness:

Attending: I couldn't find anything with the tests, your chest x-ray looks fine, your enzymes are fine and they should show if you had problems with your heart a couple of hours ago. But I want to keep you here overnight.

\section{Patient: What do they want to do?}

Attending: Probably a stress test [of the patient's heart].

Patient: Naa ... I want to go home.

Attending: We should do at least one more blood test. You can go home after the blood test comes back negative and after I call the clinic for a follow-up appointment tomorrow.

In this example, the Emergency Department physician identified the need and relevant parameters (checking for myocardial infarction, addressing the patient's previously articulated concern about hypertension) based on his biomedical knowledge of the state space and the available resources for action. More important, the physician was aware that he was accountable for making sure the patient did not suffer from an acute and severe heart problem. His professional norms suggested admitting the patient for observation overnight. To accommodate patient values, he agreed to omit admission, but only if an additional test came back negative. The decision for another test was, therefore, under the purview of the physician. Although the physician made all the decisions in this instance, by articulating his decision-making process, he allowed the patient to provide input resulting in a mutually acceptable trajectory for care.

In summary, despite a biomedical rationale for a particular choice, physician-dominated decisions may still involve and, in some cases, require coordination with patients' values. In both acute and chronic disease management, the need to create a common trajectory of care may necessitate the articulation of the physician's clinical cognition and the incorporation of the patient's perspective.

\section{Physician-Defined, Patient-Made Decisions}

In physician-defined, patient-made decisions, the physician elaborates relevant parameters, and the patient makes a final selection. Typically, the physician presents information about a limited set of options, so that the patient can make an informed choice. This decision pattern appears in the literature on shared decision making (Elwyn et al., 2012). Decisions of this type often occur when the physician's understanding of the physical constraints (e.g., pathophysiology) in the state space and properties of the potential options in the action space (e.g., efficacy, side effects) is essential, but the patient enacts the decision (e.g., taking the medication) and incurs the majority of consequences (e.g., side effects, costs). This decision pattern reflects the interaction between the physician's privileged access to biomedical information and the patient's ability to act.

In Multiple Sclerosis management, decisions that follow this pattern typically involve the selection of a course of treatment or use of complementary treatments (e.g., physical therapy). These decisions are technical, requiring physician knowledge, but involve substantial action by the patient. The distributed dynamic this creates is especially clear when the patient's norms conflict with the physician's analysis. For example, the patient in the dialogue below initially refused conventional treatment in favor of alternative medicine. What is interesting here is not merely that the patient contributed to the final decision, but that to resolve the conflict, the physician redefines the decision parameters.

Physician: I remember you saying that you decided not to do and you were saying that you were thinking more about natural 
medicine. Copaxone $\AA$ is more like a mixture of amino acids and so it's milder than Betaseron ${ }^{\circledR}$. Betaseron ${ }^{\circledR}$ is like Interferon. That's why it's a strong, little bit more synthetic I would say.

Patient: OK.

Physician: Copaxone ${ }^{\circledR}$ would be more natural. That's the reason I was just, I was changing my mind. [ . . . ] So you're comfortable with the Copaxone ${ }^{\circledR}$ or you prefer the Betaseron ${ }^{\circledR}$ medication?

Patient: Um, I read both and the Copaxone ${ }^{\circledR}$ actually sounded like, in the stuff that I read, sounded like something I'd rather do because it seemed more simple . . .

The physician begins to set up possible alternative courses of treatment and implied trade-offs by introducing a new medication (Copaxone ${ }^{\circledR}$ ) and contrasting it with a medication she previously recommended (Betaseron $\left.{ }^{\circledR}\right)$. She represents the chemical structure of the new medication as more compatible with the patient's norms for acceptable treatments (preferences for natural medicine) and presents the patient with an explicit choice of medication. The patient's selection echoes the physician's definition of the decision parameters, accepting the medication as a more "natural" alternative. By refusing a course of action initially proposed by the physician, the patient expanded the set of usual decision parameters, requiring the physician to present a new alternative in a way that aligns more closely with the patient's norms. This illustrates the inherent interdependence between the patient's action choices and the physician's clinical reasoning.

The dependence of clinical decision making on patient choice of action was also apparent in the Emergency Department. In one example, a 77-year-old patient fell and felt something "burst" in her head:

Physician: The CAT scan is showing no bleed but it's not $100 \%$ correct; $3-5 \%$ of all actual bleeds are not detected with a cat scan. The only way to be $100 \%$ sure is to do a spinal tap. [ . . ] If you ask me what I think? Is that caused by an aneurysm? No. But the lawyers and medical experts tell me to do it anyway. I can't prove it $100 \%$ in any other way. I will be honest with you: It's not easy in a 77-year old patient. $[\ldots]$

Daughter: What else can we do? What else can cause it?

Physician: Many things. [ . . . ] I suggest getting her admitted for observation and they can consult with neurosurgery if they think that's necessary. She is sitting on the fence, definitely. Do you have anything against keeping her here?

Daughter: It's up to her [looking at her mother].

Patient: [looking at her daughter]. If you think that is best for me.
In this example, the Emergency Department physician explained the dynamics of an aneurysm in the state space and gave recommendations and options for how to best react. Although the physician considered legal implications and recommended admission, he judged the probability of an aneurysm low, which allowed him to give the patient the final choice whether to stay in the hospital for further tests. Relative to the physician-dominated Emergency Department example presented earlier, this example demonstrates that part of the physician's task is to identify which physical constraints warrant physician-dominated decisions and which allow for physician-defined, patient-made decisions, which are more reflective of patient norms and values.

Despite domain differences, these examples from chronic and acute care illustrate the same pattern of distributed clinical cognition. In each case, the physician directs the dyad's attention to specific physical properties and associated potential actions, essentially mapping possible trajectories of care. The physician then interprets and discusses the desirability of these trajectories in light of patient values and norms. The patient uses the information and analysis from the physician to make a final decision. In these situations, the challenge is turning a distributed cognitive problem into a shared trajectory for reasoning about care. The physician's efficacy depends on framing decisions in a way that is mindful of the parameters that correspond to the patient's norms and values.

\section{Patient-Defined, Physician-Made Decisions}

During patient-defined, physician-made decisions, the patient guides the trajectory for clinical cognition. Hints of this pattern appear in the literature concerned with patients seeking the treatment of pain and infection (Stivers, 2002). Our analysis suggests that this pattern can be found more generally when the patient held privileged knowledge of the state space (e.g., phenomenology of symptoms, case history) and the physician held powers of action (e.g., write prescriptions or order tests). In these cases, patients used their knowledge to define the parameters of the decision either generally or as a fixed choice within a limited set of actions. The physician's role was to correlate the range of possible treatment options, biomedical aspects of the state space, and associated decision parameters outlined by the patient to make a final and informed choice. In many cases, some or all the options required action by the physician, at least in the form of a prescription. This structure places physicians in a gatekeeper role requiring them to make the final decision; a patient may prefer a particular option and set up a choice accordingly but cannot enact it without physician participation. 
In the Multiple Sclerosis clinic, patient-defined decisions typically involved the treatment of symptoms introduced by the patient. This is an area where the patient's access to experiential information about symptoms is critical. Similarly, treating symptoms typically involves judgments about comfort and functionality rather than medical necessity, so the patient's values play a larger role. The physician's role is to match the patient-defined parameters with available medical options and provide access to those options she deems appropriate. In the example below, all these elements are at work.

Patient: I had stopped (hydrocodone) at the beginning of that week unfortunately been a day, a day or two, I went the hospital. They gave me, he wrote a script for me. Ay, ay, I took the medicine as soon as could. And sure you know within 4 hours, or actually within 20 minutes I started to feel better. Within an hour my quality of life just improved dramatically ...

Physician: [Which medication helps you the best? Pain mediation?

Patient: Hydrocodone, ... Methadone seemed to work really well but then when I started to taper off of it. It was, it was the worst thing that's ever happened to me.

Physician: What happened?

Patient: Um, my body went into the worst type of shock. Just withdrawal and shock. Uh, uh, uh. I never want to take anything that my body becomes that reliant on.

Physician: The last thing you were on, how much did you take?

Patient: I brought you this. This is what, this is, this is what I've been taking. That's what I usually take. And it just depends on what the pain level is. If I can take one, I take one . . . And like I said the Requip ${ }^{\circledR}$ it didn't work. It didn't even make me sleep and uh the Cymbalta ${ }^{\circledR}$ I just I did not like the way it made me feel. And it didn't work for the pain. For some reason this pain is ...

Physician: So um yeah. So I wrote down what you respond to and I will give you some until you get to the pain clinic

This patient had Multiple Sclerosis for a long time and suffered from chronic neurological pain. He had run out of the pain medication he usually took and wanted the physician to give him a new prescription. Therefore, he used information he had about the state space and knowledge of the physician's norms to set up decision parameters intended to help the physician decide to provide the desired treatment. He began by describing his need for pain treatment as crucial, suggesting the current state required immediate action. He then described a negative withdrawal experience and his corresponding desire to avoid future dependence by minimizing his use of medication. This addressed the physician's norms that access to narcotics should be limited because of issues of abuse. Finally, he limited the options for treatment, listing several non-narcotic medications that he had tried unsuccessfully. This framed the decision narrowly for the physician: She could leave the patient in pain until he saw a pain specialist or prescribe a medication that conflicted with norms limiting narcotic use. Ultimately, she accepted the patient's implicit argument that the need to treat with a narcotic outweighed the addiction risk.

This interaction reflects the specific information and action profile that each participant had available and could contribute to negotiating an acceptable trajectory of care. The patient provided the experiential and case history information needed to support a decision and the physician had the authority to act and corresponding responsibility for judging the advisability of particular actions.

Similar cases were found in the Emergency Department when patients came in for acute exacerbations of chronic or recurrent conditions. Emergency physicians were also dependent upon the patients to provide critical information about the state space. This process was often complicated by concealed intentions as reflected in, for instance, exaggerated symptoms (e.g., the "narcotic-seeking" patient) or minimized symptoms (e.g., patients who did not acknowledge the intensity of their pain). The following patient-defined, physician-made decision provides a more subtle illustration of the dependence on patient framing, conflicting with an implicit, ultimately questionable norm that patient knowledge is not immediately relevant or needed during acute diagnostic care. A patient with a complex medical history including anemia presented at the Emergency Department reporting pain in her back and shoulder. For about two hours, the Emergency Department staff explored various possible causes including shingles and pulmonary problems. Finally, the patient indicated that the symptoms felt like she was "anemic again" and needed a transfusion. This simple framing altered the physician's cognitive trajectory surrounding the case, suspending effort to rule out a set of potentially complex problems to determining anemia as a sufficient cause for admission and terminating emergency care delivery.

In both cases, the decision was split because information about the state space and the ability to act were distributed across actors. In patient-defined, physician-made decisions, the patient has the information to identify the need for a decision but cannot or does not know how to translate this into action, whereas the physician has the knowledge of the physical constraints that allows her to 
judge the advisability of a given action and societal authorization to implement it. This distribution of knowledge and action yields a distribution of cognitive processes. Given this pattern, the efficacy of the encounter depends on the patient's ability and willingness to share experienced concerns accurately and the physician's ability to identify viable and medically justifiable options to address these concerns.

\section{Patient-Dominated Decisions}

Patient-dominated decisions occur when the patient can act independently of the physician. This pattern appears in the literature concerning patient adherence (Vermeire, Hearnshaw, Van Royen, \& Denekens, 2001). But as with patient-dominated, physician-made decisions, our analysis suggests that the patient-dominated pattern should be viewed as part of a wider continuum of shared decision making and must not be limited to the common focus on adherence. Patients independently made two major types of medical decisions: to seek care and to discontinue care. Care seeking is rarely considered in discussions of medical decision making because it takes place prior to entering the medical system. However, nearly all the patients in the Emergency Department and a few of the Multiple Sclerosis patients deliberately chose to seek care. When Emergency Department patients decide to go to urgent care, wait for a clinic appointment, or forego medical attention, they trade off the risk of missing critical treatment with the costs in money and time associated with an emergency visit. Similarly, Multiple Sclerosis patients experiencing altered symptoms make judgments about the urgency of medical needs (requiring care from an Emergency Department or through a clinical visit) and the proper provider (a primary care physician, neurologist, or other specialist). Because clinical cognition and care inherently differ depending on medical specialization, when a patient chooses a provider, they initiate a specific trajectory for both care and clinical cognition.

Patients do choose to stop acting in a way that has been previously discussed (e.g., cancel an appointment, discontinue a medication, or delay treatment). From the perspective of distributed cognition, such non-adherence reflects a failure to develop a shared trajectory for clinical reasoning during an inherently distributed task.

Most patients understand that taking action independently from the physician is considered a violation of cultural norms. As such, these decisions typically entered the conversation obliquely as reports of past behavior that influenced the current decision space. For example,

Patient: No, I don’t take Provigil® anymore.

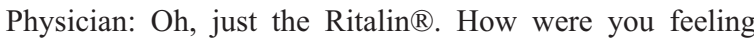
when you were taking both?

Patient: I never took both.

Physician: Oh, I thought that it was in the notes.

Patient: Oh no, no, no. As soon as I started taking — as he put me on the Ritalin $\AA$, I stopped taking Provigil ${ }^{\circledR}$ because I was scared to take them together.

This decision is presented at the beginning of the dialogue as information to include in the current state space, rather than at the end as a product of an articulated process of reasoning. After reporting the decision, the patient works backward to reveal the issues that prompted (and justified) taking a deliberate action. In the Multiple Sclerosis clinic, many patient-dominated decisions, as above, could be classified as non-adherence. But, the patients were actually engaging in self-care according to their own analysis of the decision space in the absence of a shared trajectory for clinical reasoning between patient and provider.

Given that Emergency Department physicians generally do not follow their patients, there were no similar cases of patient's choosing to modify previously agreed upon treatment regimens. However, physicians were aware that patients leaving the hospital ultimately made their own treatment decisions. Discussions surrounding patients' decisions ranged from pragmatic (e.g., unhappy patients may not disclose relevant information or may require additional attention- - "Let's get people admitted and clean up triage [ . . . I don't want to have those people $[\ldots]$ come back in three hours and we have to deal with them then.") to helpful (e.g., providing resources for patient self-care) to pejorative (e.g., a comment that a patient would "have to choose between meds and cigs") to ethical (e.g., consideration of patient preferences when deciding to treat, admit, or discharge a patient). In this sense, the patient's unique role as the final decision maker and actor was also part of the context informing decision making in the Emergency Department.

\section{Limitations}

This study is limited by constraints on data collection by observation as well as the selected settings. Our presence during data collection was apparent to physicians, patients, and family members. We view the risk of influence on physicians as minimal due to the critical nature of their professional responsibilities. We also view the risk of influence on patients as minimal, due to the critical nature of the concerns that motivated their quest for medical care. Although the use of two different types of care 
suggests a degree of generalizability, in other medical settings specific aspects of the decision space may influence the distribution of cognitive processes in ways that are not anticipated by this study. For instance, the technical foundations of decisions in an Intensive Care Unit might alter the way that patients and physicians approach decision making or the feasibility of all patterns.

Given that we used two existing data sets, we were not able collect data in a motivated fashion to confirm the developing theory or to verify that data saturation was achieved. However, the relatively large corpus available for analysis and the convergence of two data sets suggests that the data were sufficient to support the findings presented. In retrospect, the observed decision patterns represent a logical spectrum of the distribution of decision-making responsibility, even if they may not include all possible variations.

Finally, the focused analysis of decision making based on the Decision Space Model does not substitute for a holistic ethnography of medical decision making in the observed domains of the health care system. That is, the model offers a way to conceptualize, categorize, and discuss the influences on decision making, but does not elucidate the specific socio-cultural and technical processes that create these influences in chronic and acute care. Future research is needed to explore the meaning of and role that specific chronic illnesses such as Multiple Sclerosis, or acute health problems such as heart attacks or strokes, play in structuring decisionmaking situations.

\section{Conclusion}

This study suggests a reconceptualization of medical decision making in terms of the distributed cognition paradigm. We began with the Decision Space Model to clarify the relationship between physical and social influences on decision making. Using concrete instances, we demonstrated that both patients and physicians consider these influences when identifying the need for a decision, searching for relevant decision parameters, making actionable decisions, and developing satisfactory care trajectories. Depending on the specific access of patients and physicians to information and resources for action, we identified four different patterns of decision making in both acute and chronic care settings. These patterns form a continuum of shared decision making, uniting the traditional decision-making researcher's focus on physicianled decisions and patient participation with the practitioner's concern for assessing patients' needs and problems of adherence. In other words, these patterns help to integrate issues raised in different literatures into a more general framework fusing individual and shared decision making.
The physician-dominated decision, prevalent in the cognitively oriented decision-making literature was only one of several observed decision patterns. Moreover, even in these situations, some instances required knowledge, or at least consideration, of the patient's interpretation of the decision space - their norms, values, and opportunities to act-for effective and justifiable decisions. Other observed patterns included split-decisions where one party specified the parameters of the decision and the other party made a final decision and decisions that were made only by patients. The consistent theme across the identified decision patterns is that medical decisions are predicated on the division of information and the ability to act. Thus, the participation of both physicians and patients in decision making is not just socially desirable, as suggested by the shared decision-making paradigm, but the inevitable result of the distribution of clinical cognition.

This study yielded three key findings. First, medical decision making is fundamentally distributed between physicians and patients; this is true not only in chronic disease management but also occurs in acute care. Second, the ways in which actors conceptualize and evaluate physical, personal, and socio-cultural constraints on the decision space can vary substantially, emphasizing the need to negotiate problems and solutions and to create shared trajectories for clinical cognition. Effectively, each actor's understanding of the decision space becomes a constraint on the state space of every other actor. Finally, the different patterns of shared decision making we identified suggest that in natural environments, decision making is a temporally extended process including the identification of a decision point, the definition and assessment of relevant parameters, and the final choice, which represents but one episode in a care trajectory. The distribution and organization of these tasks depend on actors' understanding of and physically and socially constrained capacity to act within the decision space.

These findings have implications for both theoretically and clinically oriented work. First, the Decision Space Model unites cognitive-behavioral processes, physical constraints, and socio-cultural considerations. In examples from the management of both chronic and acute care, the interplay of informational, physical, and sociocultural perspectives became evident in nearly every interaction. Even when physicians were making highly technical decisions, they accounted for socio-cultural constraints in addition to purely biomedical and resource considerations. From a theoretical perspective, this suggests that experimental research on medical cognition, most of which is phrased in terms of probabilistic biomedical concerns, fails to account for a substantial portion of the decision process. It also suggests a need for more research into the cognitive processes underlying 
shared decision making and for medical education to train professionals how to negotiate the interplay of social, resource, and biomedical considerations.

Second, the Decision Space Model suggests that decision making spans actors' conceptualization of the environment and their opportunities and requirements for action. Analysis based on this assumption helped to identify a multi-stage decision process involving the identification of decision points, refinement of decision parameters, and a final decision. A focus on the division of available information and action opportunities between actors underscores the pervasive distribution of cognitive processes along this temporal trajectory. On a theoretical level, the observed dynamics between state and action spaces suggest that decision-making research needs to be sensitive to more than just final decisions. More research is needed to understand how the relationship between state and action space triggers the need for a decision and defines the parameters to be considered during the final decision.

Finally, the expanded Decision Space Model incorporates contributions from multiple actors. When information, cognition, and action are distributed, actors must inform one another about relevant influences (including implicit norms and values) and clearly communicate decision parameters to allow for common ground (e.g., Wilkes-Gibbs \& Clark, 1992) and coherent decisions. This finding highlights the core contributions patients make to clinical cognition, including patterns of decision making that are patient led, which have typically been overlooked or dismissed as "non-adherence." Unless physicians and patients transform the distributed task into a shared cognitive trajectory, physicians may misdiagnose symptoms or patients may be unable (or unwilling) to comply with prescribed treatments. Thus, on a clinical level, medical staff should be trained and patients should be asked to communicate their understanding of state and action space so that mismatches can be identified early. Our findings further suggest that studying patients' reasoning processes - including prior to entering the health care system - is important to better understand the extent to which decisions are necessarily shared and that limiting research to medical decision making in the doctor's office risks reinforcing a limited paternalistic model.

In summary, the results suggest a need for both extension and integration of current models of medical decision making. First, a better understanding of shared decision making will require consideration of the role of distributed medical, physical, and social factors in physicians' and patients' reasoning. It also will require an extended focus on processes related to the identification of decision needs and the search for decision parameters, in addition to those involved in the final decision. Second, the identified decision-making patterns unite researchers' and clinicians' concerns with a common underlying framework of distributed medical decision making. By expanding the Decision Space Model with the observed patterns of distributed cognition, the future research agenda is substantially clarified: The four dimensions of the decision space pose different specific possibilities of individual and shared responsibility for decision making to investigate.

From an applied perspective, the interdependence of physician and patient cognition suggests that a goal for improving clinical decisions should not only be to instruct physicians to engage intentionally in shared decision making, that is, a change in attitudes. Training should rather focus on helping physicians and patients understand and improve upon the ways they already adapt their cognition to accommodate the division of information and resources for action. This way both parties may be empowered to better coordinate information, cognition, and action and thereby improve the quality of care, adherence, and treatment outcomes.

\section{Acknowledgments}

The authors thank Roxana Wales and David Messinger for sharing their perspectives on anthropological methods and graphics design expertise. Helen Klein and John Flach contributed to the intellectual environment that fostered this research. Our analysis was only possible with the generous participation of physicians and patients.

\section{Declaration of Conflicting Interests}

The authors declared no potential conflicts of interest with respect to the research, authorship, and/or publication of this article.

\section{Funding}

The authors disclosed receipt of the following financial support for the research, authorship, and/or publication of this article: This work was partially supported by the NSF IGERT program, award DGE-0504438: John Flach, PI.

\section{Note}

1. The validity of these categories was independently supported by a statistical factor analysis of another data set collected in this setting, which found an equivalent distinction-cues related to goal establishment and goal enactment - to describe physician information seeking behavior (Robinson 2011).

\section{References}

Barber, N. (2002). Should we consider non-compliance a medical error? Quality \& Safety in Health Care, 11, 81-84. doi:10.1136/qhc.11.1.81

Bernabeo, E., \& Holmboe, E. S. (2013). Analysis \& commentary: Patients, providers, and systems need to acquire 
a specific set of competencies to achieve truly patientcentered care. Health Affairs, 32, 250-258. doi:10.1377/ hlthaff. 2012.1120

Broadstock, M., \& Michie, S. (2000). Processes of patient decision making: Theoretical and methodological issues. Psychology \& Health, 15(2), 191-204. doi: 10.1080/08870440008400300

Cameron, L., Leventhal, E. A., \& Leventhal, H. (1993). Symptom representations and affect as determinants of care seeking in a community-dwelling, adult sample population. Health Psychology, 12, 171-179. doi:10.1037/02786133.12.3.171

Corbin, J. M. (1998). The Corbin and Strauss Chronic Illness Trajectory model: An update. Scholarly Inquiry for Nursing Practice, 12(1), 33-41.

Cowley, S. J., \& Vallée-Tourangeau, F. (2013). Cognition beyond the brain. London: Springer.

Croskerry, P. (2009). A universal model of diagnostic reasoning. Academic Medicine, 84, 1022-1028. doi:10.1097/ ACM.0b013e3181ace703

Custers, E. J. (2013). Medical education and cognitive continuum theory: An alternative perspective on medical problem solving and clinical reasoning. Academic Medicine, 88 , 1074-1080.

Elwyn, G., Frosch, D., Thomson, R., Joseph-Williams, N., Lloyd, A., Kinnersley, P., . . . Barry, M. (2012). Shared decision making: A model for clinical practice. Journal of General Internal Medicine, 27, 1361-1367. doi:10.1007/ s11606-012-2077-6

Elwyn, G., Lloyd, A., May, C., van der Weijden, T., Stiggelbout, A., Edwards, A., . . . Epstein, R. (2014). Collaborative deliberation: A model for patient care. Patient Education \& Counseling, 97, 158-164. doi:10.1016/j.pec.2014.07.027

Engeström, Y., Engeström, R., \& Kerosuo, H. (2003). The discursive construction of collaborative care. Applied Linguistics, 24, 286-315.419. doi:10.1093/applin/24.3.286

Epstein, R. M. (2013). Whole mind and shared mind in clinical decision-making. Patient Education \& Counseling, 90, 200-206. doi:10.1016/j.pec.2012.06.035

Feufel, M. A. (2009). Bounded rationality in the emergency department (Doctoral dissertation, Wright State University, Dayton, $\mathrm{OH}$ ). Retrieved from https://etd. ohiolink.edu/pg_10?0::NO:10:P10_ACCESSION NUM:wright 1249241698

Feufel, M. A., Robinson, F. E., \& Shalin, V. L. (2011). The impact of medical record technologies on collaboration in emergency medicine. International Journal of Medical Informatics, 80(8), e85-e95.

Ghane, A., Huynh, H. P., Andrews, A. M., Tabuenca, A., \& Sweeny, K. (2014). The relative importance of patients' decisional control preferences and experiences. Psychology \& Health, 29(10), 1-29. doi:10.1080/088704 46.2014.911873

Hazlehurst, B., McMullen, C. K., \& Gorman, P. N. (2007). Distributed cognition in the heart room: How situation awareness arises from coordinated communications during cardiac surgery. Journal of Biomedical Informatics, 40, 539-551. doi:10.1016/j.jbi.2007.02.001
Hibbard, J. H., \& Greene, J. (2013). What the evidence shows about patient activation: Better health outcomes and care experiences; fewer data on costs. Health Affairs, 32, 207214. doi:10.1377/hlthaff.2012.1061

Hutchins, E. (1995). Cognition in the Wild. Boston: MIT Press.

Klein, G., \& Wright, C. (2016). Macrocognition: From theory to toolbox. Frontiers in Psychology, 7(54), 1-5.

Kleinman, A. (1980). Patients and healers in the context of culture: An exploration of the borderland between anthropology, medicine, and psychiatry (Vol. 3). Oakland: University of California Press.

Légaré, F., \& Witteman, H. O. (2013). Shared decision making: Examining key elements and barriers to adoption into routine clinical practice. Health Affairs, 32, 276-284. doi:10.1377/hlthaff.2012.1078

Lippa, K. D., \& Shalin, V. L. (2015, July). Stepping up to the blackboard: Distributed cognition in doctor-patient interactions. Paper presented at CogSci, Pasadena, CA.

Malone, T. W., \& Crowston, K. (1994). The interdisciplinary study of coordination. ACM Computing Surveys, 26, 87119. doi: $10.1145 / 174666.174668$

March, J. G., \& Olsen, J. P. (2006). The logic of appropriateness. In M. Rein, M. Moran \& R. E. Goodin (Eds.), Handbook of public policy (pp. 689-708). Oxford: Oxford University Press.

Marewski, J. N., \& Gigerenzer, G. (2012). Heuristic decision making in medicine. Dialogues in Clinical Neuroscience, 14, 77-89.

Phang, S. H., Ravani, P., Schaefer, J., Wright, B., \& McLaughlin, K. (2015). Internal Medicine residents use heuristics to estimate disease probability. Canadian Medical Education Journal, 6(2), e71-e77.

Reyna, V. F., \& Lloyd, F. J. (2006). Physician decision-making and cardiac risk: Effects of knowledge, risk perception, risk tolerance, and fuzzy processing. Journal of Experimental Psychology: Applied, 12, 179-195.

Robinson, E. (2011). The role of deliberate behavior in expert performance: The acquisition of information gathering strategy in the context of emergency medicine. Unpublished master's thesis, Wright State University, Dayton, $\mathrm{OH}$.

Roter, D., \& Hall, J. (2006). Doctors talking with patients/ patients talking with doctors: Improving communication in medical visits. Westport, CT: Praeger.

Schwartz, A., \& Elstein, A. S. (2009). Clinical problem solving and diagnostic decision making: A selective review of the cognitive research literature. In J. A. Knottnerus \& F. Buntinx (Eds.), The evidence base of clinical diagnosis: Theory and methods of diagnostic research (pp. 237-255). London: Blackwell.

Shalin, V. L., \& Bertram, D. A. (1996). Functions of expertise in a medical intensive care unit. Journal of Experimental \& Theoretical Artificial Intelligence, 8, 209-227.

Simon, H. A., \& Newell, A. (1971). Human problem solving: The state of the theory in 1970. American Psychologist, 26, 145-159.

Stivers, T. (2002). Participating in decisions about treatment: Overt parent pressure for antibiotic medication in pediatric encounters. Social Science \& Medicine, 54, 1111-1130. 
Strauss, A. L., \& Corbin, J. (2008). Basics of qualitative research: Techniques and procedures for developing grounded theory. New York: Sage.

Strauss, A. L., Fagerhaugh, S., Suczek, B., \& Wiener, C. (1995). Social organization of medical work. Chicago IL: University of Chicago Press.

Thille, P. H., \& Russell, G. M. (2010). Giving patients responsibility or fostering mutual response-ability: Family physicians' constructions of effective chronic illness management. Qualitative Health Research, 20, 1343-1352. doi:10.1177/1049732310372376

Vermeire, E., Hearnshaw, H., Van Royen, P., \& Denekens, J. (2001). Patient adherence to treatment: Three decades of research. Journal of Clinical Pharmacy and Therapeutics, 26, 331-342.

Wilkes-Gibbs, D., \& Clark, H. C. (1992). Coordinating beliefs in conversation. Journal of Memory and Language, 31, 183-194.

\section{Author Biographies}

Katherine D. Lippa defended her dissertation in spring 2016 at Wright State University. Her research interests include patient cognition, doctor-patient interactions, and the development of expertise.

Markus A. Feufel is a research scientist at CharitéUniversitätsmedizin Berlin. His main interest is on decision making under uncertainty and risk communication as a prequisite for informed decision making.

Eric Robinson is currently completing his $\mathrm{PhD}$ at Wright State University. His research interests include how experts gather information from the world and the interaction between deliberative and automatic processes in expert behavior.

Valerie L. Shalin is an associate professor in the Department of Psychology and member of the Ohio Center of Excellence in Knowledge Enabled Computing at Wright State University. 